\title{
OSIRIS - An online scintillator radiopurity monitor for the JUNO experiment
}

\author{
Moritz Cornelius Vollbrecht for the JUNO collaboration ${ }^{1,2}$ \\ ${ }^{1}$ Forschungszentrum Jülich GmbH, Nuclear Physics Institute IKP-2 \\ Wilhelm-Johnen-Straße, Jülich, Germany \\ ${ }^{2}$ III. Physikalisches Institut B, RWTH Aachen University \\ Otto-Blumenthal-Straße, Aachen, Germany
}

E-mail: m.vollbrecht@fz-juelich.de

The Jiangmen Underground Neutrino Observatory (JUNO), currently under construction in China, will be the first multi-kton liquid scintillator detector and has a vast potential for new insights into several fields of neutrino and astroparticle physics. To reach its design sensitivity for detecting reactor and solar neutrinos, a radiopure liquid scintillator is required. For IBD measurements, a radiopurity of $10^{-15} \mathrm{~g} / \mathrm{g}$ is needed for both ${ }^{238} \mathrm{U}$ and ${ }^{232} \mathrm{Th}, 10^{-16} \mathrm{~g} / \mathrm{g}$ for solar measurements.

The Online Scintillator Internal Radioactivity Investigation System (OSIRIS) allows an on-line radiopurity evaluation of the scintillator during the JUNO detector filling over several months. The design of OSIRIS is optimized for tagging ${ }^{214} \mathrm{Bi}^{214} \mathrm{Po}$ and ${ }^{212} \mathrm{Bi}^{212} \mathrm{Po}$ coincidence decays in the decay chains of ${ }^{238} \mathrm{U}$ and ${ }^{232} \mathrm{Th}$, respectively. OSIRIS will also be able to monitor the ${ }^{14} \mathrm{C}$ and ${ }^{210}$ Po levels in the scintillator.

To achieve its goals, OSIRIS features a 20 ton liquid scintillator target monitored by 76 intelligent photomultiplier tubes (iPMTs). In this novel design, each iPMT consists of a PMT and its readout electronics mounted on its back. Each hit causing these electronics to trigger is sent to the DAQ as a digitized PMT pulse. A single computer (EventBuilder) is sufficient to combine the data stream into events for further analysis.

For the timing and charge calibration of the detector, two optical systems (LED- and LASER-based) are employed. The energy and position calibration of OSIRIS is performed with height-adjustable radioactive sources within the liquid scintillator. These sources cover the crucial energy range for the detection of Bi-Po signals between $0.66 \mathrm{MeV}$ to $2.5 \mathrm{MeV}$.

\footnotetext{
*** The European Physical Society Conference on High Energy Physics (EPS-HEP2021), ***

***26-30 July $2021 * * *$

*** Online conference, jointly organized by Universität Hamburg and the research center DESY ***
} 


\section{Jiangmen Underground Neutrino Observatory (JUNO)}

The Jiangmen Underground Neutrino Observatory (JUNO) is currently under construction in Southern China. After the completion of its construction in 2022, JUNO is expected to have a vast potential for new insights into various topics in (astro-)particle physics. The main goal of JUNO will be the determination of the neutrino mass hierarchy via the measurement of reactor antineutrino oscillation patterns at a baseline of $53 \mathrm{~km}$. Being the first multi-kton liquid scintillator detector, it features a scintillator target with a diameter of $35 \mathrm{~m}$, instrumented with 17612 20"-PMTs and 25600 3"-PMTs. This results in a total optical coverage of $77.9 \%$ and an expected energy resolution of $3 \%$ at $1 \mathrm{MeV}[1]$.

In order to reach JUNO's design sensitivity, a radiopure liquid scintillator target is needed. Inverse Beta Decay (IBD) measurements with JUNO require a radiopurity of $10^{-15} \mathrm{~g} / \mathrm{g}$ for both ${ }^{238} \mathrm{U}$ and ${ }^{232} \mathrm{Th}$, a radiopurity of $10^{-16} \mathrm{~g} / \mathrm{g}$ is needed for solar measurements [2].

\section{Online Scintillator Internal Radioactivity Investigation System (OSIRIS)}

The Online Scintillator Internal Radioactivity System (OSIRIS), shown in Fig. 1, allows an online radiopurity evaluation of the scintillator during the JUNO detector filling that lasts several months. By measuring the radiopurity of the liquid scintillator, air leaks and malfunctions in the liquid scintillator purification chain can be detected. For this purpose, OSIRIS features a 20 ton liquid scintillator target in a cylindrical acrylic vessel. The design of OSIRIS is optimized for tagging ${ }^{214} \mathrm{Bi}^{2}{ }^{214} \mathrm{Po}$ and ${ }^{212} \mathrm{Bi}^{-212} \mathrm{Po}$ fast coincidence decays in the decay chains of ${ }^{238} \mathrm{U}$ and ${ }^{232} \mathrm{Th}$, respectively [2]. Other measurands include the levels of ${ }^{14} \mathrm{C}$ or ${ }^{210} \mathrm{Po}$ for an even more detailed radiopurity analysis.

During normal operation of OSIRIS, the so-called continuous mode will be used. In this mode, a fraction of the liquid scintillator stream from the purification plants will pass through OSIRIS at a rate of about 1 ton per hour [2]. While the liquid scintillator moves through the detector, its radiopurity is constantly monitored in order to facilitate fast reactions on events contaminating the liquid scintillator.

The other operation mode of OSIRIS is called batch mode. Here, a batch of liquid scintillator is stored inside the acrylic vessel and analysed for much longer timescales, e.g. for a month. The measuring time strongly depends

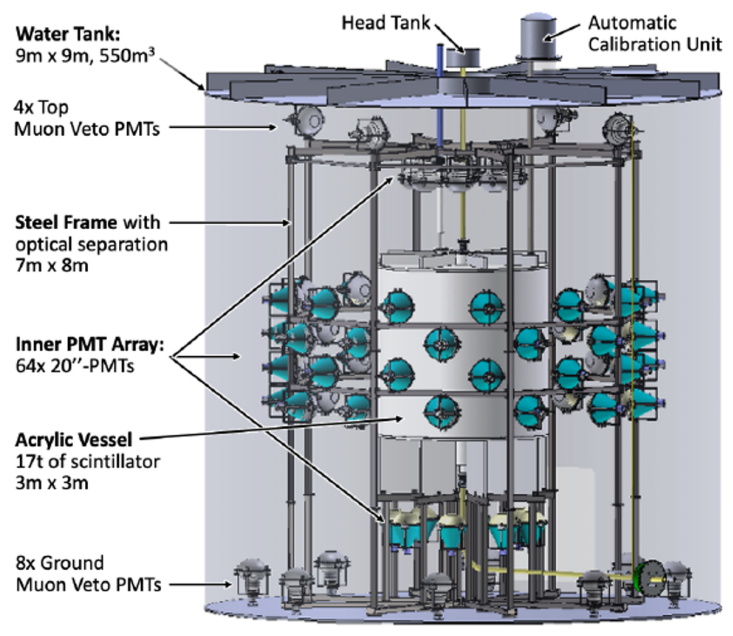
on the initial Radon contamination, see also Fig. 2. The batch mode will be mainly used during the calibration phase of OSIRIS to as-

Figure 1: Overview of the OSIRIS detector. [2] sess the detector performance and fine-tune the measurement devices distributed within the detector. 


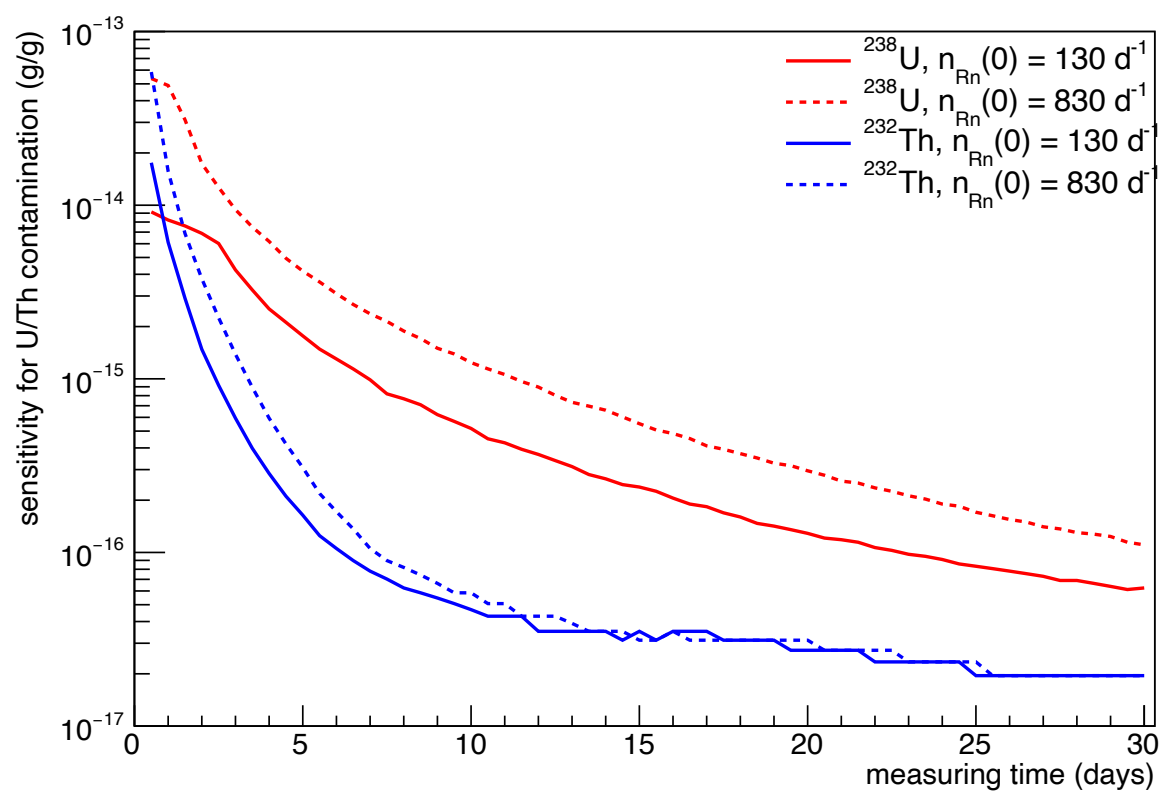

Figure 2: Contamination sensitivities against measuring time for ${ }^{238} \mathrm{U} /{ }^{232} \mathrm{Th}$ in batch mode operation [2]. The time needed to verify the LS radiopurity requirements varies between a few days and a month, depending on the initital radon contamination (dashed / solid lines).

\section{Intelligent PMTs (iPMTs)}

To achieve its goals, OSIRIS features an array of 76 intelligent photomultiplier tubes (iPMTs). In this novel design, each iPMT consists of a Hamamatsu-20"-PMT with the readout electronics mounted directly on its back. A single CAT5eEthernet-cable is sufficient to deliver power via Power-overEthernet (PoE) and to transfer data to the DAQ.

The highly configurable readout chip VULCAN from ZEA-2 of Forschungszentrum Jülich digitizes signals with a sample rate of $500 \mathrm{MS} / \mathrm{s}$. The samples are passed to a XilinxZynq-SoC with an embedded FPGA. If a sample crosses the trigger threshold, the SoC creates a readout window around this point in time. This window is then sent to the DAQ via Ethernet.

The FPGA allows the implementation of waveform reconstruction algorithms on the PMT itself for immediate online analyses, hence the term "intelligent". A sketch of the full iPMT assembly is shown in Fig. 3.

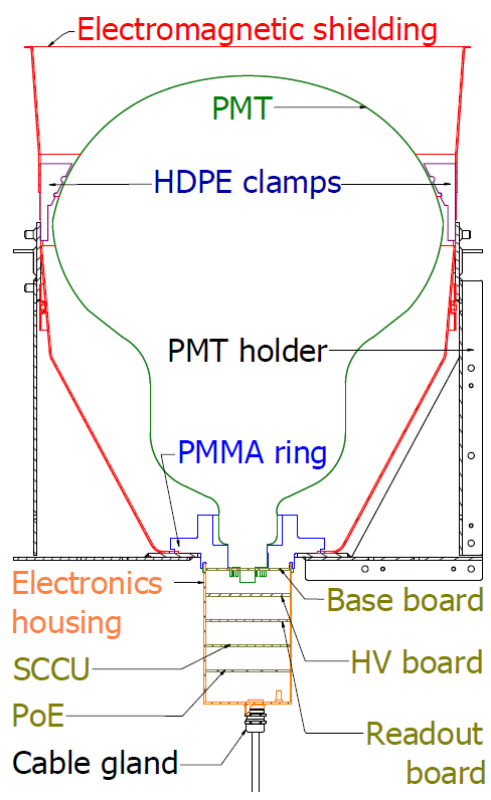

Figure 3: Crosssection of the iPMT assembly. 


\section{Data acquisition and RunControl}

The data acquisition processes the data stream that comes from the iPMTs and is condensed by a series of network switches to a single 10Gbit-connection. The data processing is performed on a single high-perfomance consumer computer, the "EventBuilder". The Eventbuilder sorts the incoming data packages corresponding to the timestamps imbedded in the data format, performs coincidence searches for different scenarios and writes relevant data to storage. For this purpose, a multithreaded software framework is used to achieve high data throughputs as well as high flexibility concerning code modifications. A schematic of the software layout is shown in Fig. 4.

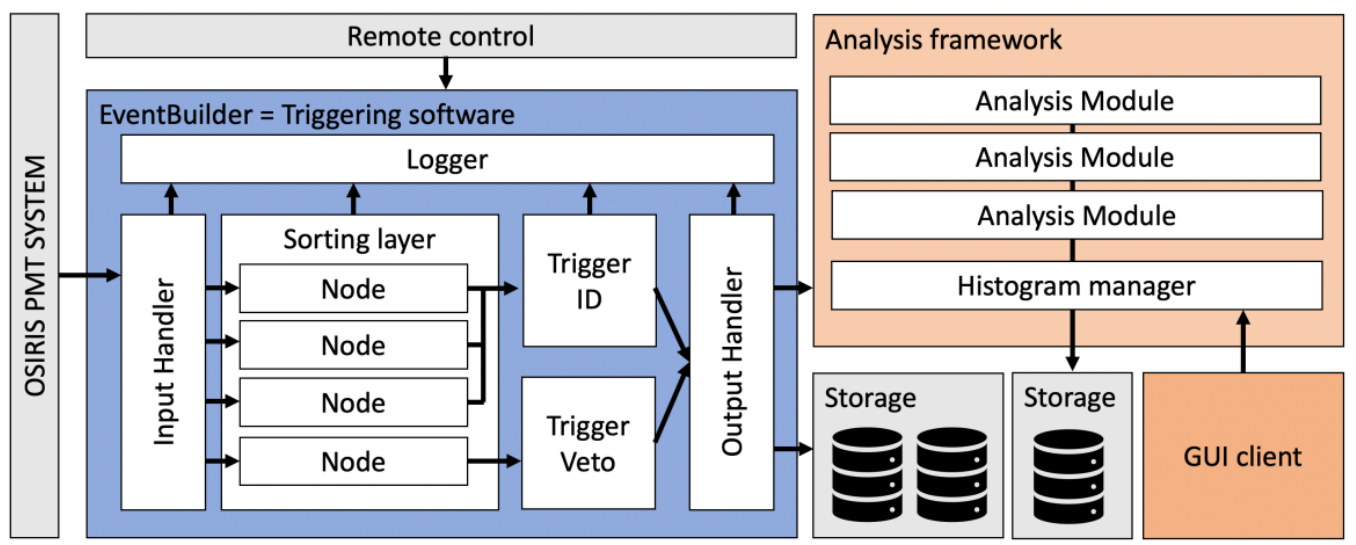

Figure 4: Data acquisition schematic. [2]

The RunControl is used to start and control the different data acquisition runs as well as to manage the different subsystems within the detector. It also offers user interfaces for online analysis of the incoming data in combination with overview possibilities for operators to spot and evaluate problems during the detector operation.

\section{Calibration systems}

For the calibration of OSIRIS, multiple systems are employed. The first system uses a refurbished Automatic Control Unit (ACU) of the Daya Bay experiment, an inside view of the ACU is shown in Fig. 5. The ACU is used for the calibration of PMT timing and charge as well as energy and vertex reconstructions. For these tasks, the ACU features three independent wheels which can be used to lower capsules directly into the liquid scintillator target along an off-center vertical axis.

The first capsule holds ${ }^{137} \mathrm{Cs},{ }^{65} \mathrm{Zn}$ and ${ }^{60} \mathrm{Co}$ sources with a combined activity of several $\mathrm{kBq}$ in an energy range between 0.66 and $2.5 \mathrm{MeV}$. The gammas emitted by these sources cover the energy range crucial for detecting Bi-Po-signals. The second capsule stores a ${ }^{40} \mathrm{~K}$ source emitting $1.46 \mathrm{MeV}$ gammas with an activity of $<1 \mathrm{~Bq}$. Under normal operation, this capsule stays within the detector volume and is used as a continuous monitor of the liquid scintillator properties. The third capsule employs a $435 \mathrm{~nm}$-LED for the PMT timing and charge calibration. The LED is powered by a LED-driver-circuit, leading to pulse durations of about $5 \mathrm{~ns}$ (FWHM) at a pulse rate of up to 3 kHz. [2] 


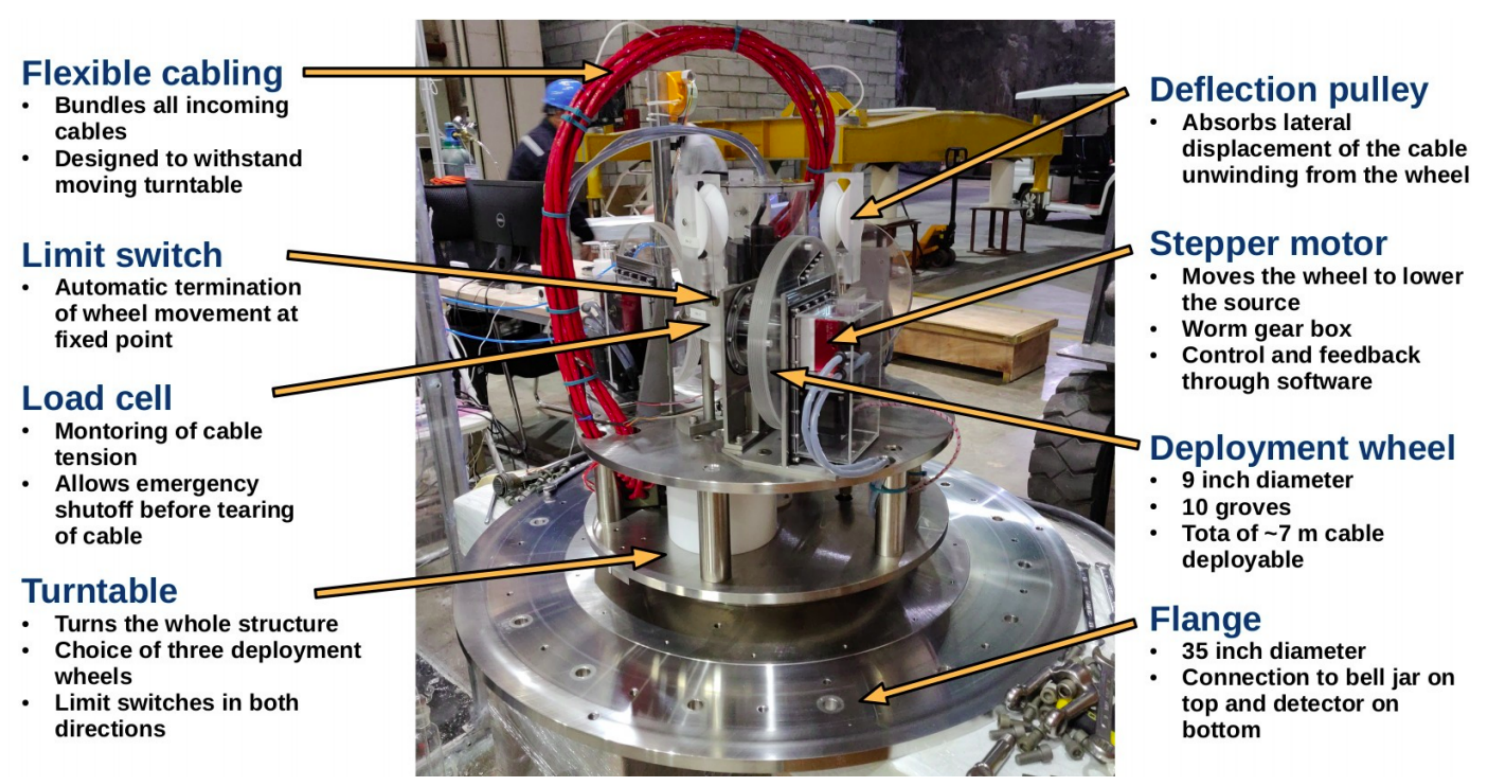

Figure 5: View inside the open ACU.

As the exact position of the capsules is critical for the calibration, the ACU records its positions via rotary encoders. However, a seperate IR-CCD camera system is installed within OSIRIS to verify the positioning calculated by the ACU, hereby adding redundancy to the determination of the capsule positions.

The second system used to calibrate OSIRIS is a laser system, distributing light with a wavelength of $420 \mathrm{~nm}$ by optical fibres and diffusors within OSIRIS. Offering light pulses with $80 \mathrm{ps}$ length at a maximum repetition rate of up to $20 \mathrm{MHz}$ [2], it is employed to calibrate PMT charge and timing calibration as a redundant system to the ACU.

The expected durations for the calibration runs depend on the stage of operation OSIRIS is in. During construction and after changes, longer calibration runs are expected to gain as much knowledge as possible about the detector behaviour. Otherwise, shorter weekly calibration runs with a few hours duration are foreseen to directly assess slower changes in the detector's performance.

\section{References}

[1] A. Abusleme et al (13 Mar 2021) „JUNO Physics and Detector“, to appear in Progress in Particle and Nuclear Physics, arXiv:2104.02565

[2] A. Abusleme et al. (31 Mar 2021), " The Design and Sensitivity of JUNO‘s scintillator radiopurity pre-detector OSIRIS“, accepted by European Journal C, arXiv:2103.16900 